\title{
An Evaluation of a Multidisciplinary Pediatric Behavioral Health Medication Initiative Workgroup's Interventions on Medication Prescribing in a Population of Medicaid Patients
}

\author{
Ashley N. Chiara, PharmD, MBA; Neha Kashalikar, PharmD; Thomas C. Pomfret, PharmD, MPH, BCPS; \\ Kimberly Lenz, PharmD; Donna M. Faber, PharmD, BCPS, BCPP; Joel Goldstein, MD; \\ Karen M. Clements, ScD; Michael C. Angelini, MA, PharmD, BCPP; Patricia Leto, PharmD; \\ and Paul L. Jeffrey, PharmD
}

\begin{abstract}
BACKGROUND: In response to concerns surrounding pediatric behavioral health medication prescribing, the Massachusetts Medicaid Pharmacy Program implemented a Pediatric Behavioral Health Medication Initiative (PBHMI), proactively requiring prior authorization for specific behavioral health medications and combination regimens. A multidisciplinary therapeutic class management (TCM) workgroup retrospectively reviews complex cases and conducts prescriber outreach to encourage evidence-based practices in Massachusetts.
\end{abstract}

OBJECTIVE: To evaluate recommendation outcomes of telephonic peer-topeer consultations conducted by the PBHMI TCM workgroup by assessing the percentage of accepted, modified accepted, or rejected recommendations, as well as prescriber satisfaction with consultation.

METHODS: This retrospective evaluation reviewed PBHMI TCM workgroup cases with completed peer-to-peer consultations from September 1, 2015, to August 28, 2016. The proportion of medication interventions (e.g., medication changes, dose reductions, and elimination of polypharmacy within or across behavioral health medication classes) accepted, modified accepted, or rejected were assessed based on pharmacy claims data and prior authorization resubmission, following a peer-to-peer consultation. The medication class and prescriber type were categorized in relation to the acceptance, modified acceptance, or rejection outcomes. Satisfaction with the TCM workgroup process was evaluated with an anonymous survey offered to prescribers who participated in prescriber outreach.

RESULTS: A total of 70 cases requiring a peer-to-peer consultation by a TCM workgroup child/adolescent psychiatrist had a completed outreach attempt during the evaluation period. Peer-to-peer consultations resulted in a recommendation acceptance rate of $31.4 \%(22 / 70)$, modified acceptance rate of $44.3 \%(31 / 70)$, and a rejection rate of $24.3 \%(17 / 70)$. Recommendations made during a peer-to-peer consultation were rejected by $30 \%(12 / 40)$ of child/adolescent psychiatrists compared with $16.7 \%$ $(5 / 30)$ of nonchild/adolescent psychiatrists with completed peer-to-peer consultations $(P=0.43)$. Antipsychotics were most frequently recommended for regimen changes. All recommendations pertaining to a benzodiazepine were accepted by the prescriber. Results of an anonymous prescriber survey assessing satisfaction with the peer-to-peer consultation process exhibited variable responses among individual prescribers.

CONCLUSIONS: The small sample size in this observational evaluation and lack of a defined control group prevented direct associations between the endpoints and outcomes. Further research is required to determine if prescriber specialty and medication class may be influencing factors on recommendation acceptance.

J Manag Care Spec Pharm. 2018;24(8):746-51

Copyright $\odot 2018$, Academy of Managed Care Pharmacy. All rights reserved.

\section{What is already known about this subject}

Diagnosis of psychiatric conditions, as well as the use of behavioral health medications in a pediatric population, has been rapidly increasing over the past decade, particularly in children with Medicaid and children in foster care.

Despite clinical advances in the treatment of psychiatric conditions, many pediatric patients do not receive adequate evaluation and treatment services, while others may be overdiagnosed and treated with inappropriate medications.

Increased use of antipsychotic medications, antipsychotic polypharmacy, or antipsychotics used in combination with other behavioral health medications predisposes pediatric patients to serious metabolic side effects and other associated risks, including increased likelihood of pharmacokinetic and pharmacodynamic interactions, potential for cumulative drug-induced toxicities, and the need for additional interventions to control side effects.

\section{What this study adds}

The development and implementation of a multidisciplinary Pediatric Behavioral Health Medication Initiative therapeutic class management (PBHMI TCM) workgroup can serve as a proactive approach for identifying complex pediatric cases involving polypharmacy and other potential safety concerns.

Further evaluation with a larger study population is warranted to determine if peer-to-peer outreach is associated with evidencebased therapeutic optimization

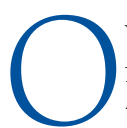
ver the past 2 decades, following the development of new treatment options and higher rates of diagnosis, there has been a considerable increase in the number of behavioral health medications prescribed to treat pediatric psychiatric disorders. Comer et al. (2010) reported that the percentage of doctor visits involving psychotropic medications, specifically for children, increased 75\% from 1996 to 2007. ${ }^{1}$ Despite clinical advances in the treatment of psychiatric conditions, the American Psychological Association and U.S. Government Accountability Office report many pediatric patients may not receive adequate therapy, while others may be 
overdiagnosed and treated with medications that may be inappropriate. ${ }^{2-6}$ Recent studies suggest that combinations of psychotropic drugs in the pediatric population increased between two- to seven-fold over the past decade, with particularly high occurrence of psychotropic polypharmacy in children enrolled in Medicaid and foster care..$^{7-12}$

Increased use of behavioral health medications or combinations of behavioral health medications predisposes pediatric patients to serious metabolic side effects including weight gain, glucose intolerance, and type 2 diabetes. ${ }^{8,14}$ Polypharmacy may also result in the increased likelihood of pharmacokinetic interactions, potential for cumulative drug-induced toxicities, and the need for additional interventions to control side effects. Patient regimens exhibiting behavioral health polypharmacy are at higher risk of adverse medical outcomes, increased financial burden, and greater medication burden. ${ }^{12-13}$

In response to these concerns, the Massachusetts Medicaid (MassHealth) Pharmacy Program, in collaboration with the Department of Children and Families and the Department of Mental Health (DMH), implemented a Pediatric Behavioral Health Medication Initiative (PBHMI) in November 2014. The clinical prior authorization (PA) criteria for the PBHMI is evidence based and was developed in collaboration with the DMH Expert Workgroup Advisory Board.

A multidisciplinary customized PBHMI therapeutic class management (TCM) workgroup was created to retrospectively review complex cases. The team includes child/adolescent psychiatrists, pharmacists, and a social worker. The PBHMI TCM workgroup interventions include telephonic prescriber outreach by a TCM workgroup child/adolescent psychiatrist to encourage evidence-based practices, opportunities for regimen simplification, and/or member referral to a behavioral health program that assists with care integration. Regimen simplification may include reducing polypharmacy or medication dose reduction. Interventions, subject to a clinician's judgment, are customized based on pharmacy and medical claims data.

The primary objective of this retrospective analysis was to identify, following each peer-to-peer consultation, the percentage of acceptance, modified acceptance, or rejection of medication interventions (e.g., medication changes, dose reductions, decrease in the number of medications prescribed, and/or elimination of polypharmacy within or across behavioral health medication classes) based on subsequent pharmacy claims data. Secondary goals included comparing the type of prescriber and the distribution of medication class discussed during the peer-to-peer consultation. Overall satisfaction with the telephonic peer-to-peer consultation process was assessed via an anonymous survey administered to prescribers who participated in an outreach call. The effect of the PBHMI TCM workgroup has not been previously evaluated.

\section{Methods}

\section{Population Selection}

This initiative proactively requires PA for specific medications or combinations of behavioral health medications prescribed to pediatric members. Member cases with a completed telephonic peer-to-peer consultation by a child/adolescent psychiatrist were included in the evaluation. Criteria for TCM workgroup discussion included cases that prescribed 5 or more medications and regimens with high-risk medications (i.e., lithium, valproate, tricyclic antidepressant, benzodiazepine, antipsychotic polypharmacy, or antipsychotic use in children aged less than 13 years). Members included in the analysis were aged less than 18 years at the time of TCM review and maintained active MassHealth Pharmacy coverage through the evaluation period. Exclusion criteria included interruptions in MassHealth coverage or third-party insurance coverage during the evaluation period. This analysis was reviewed by both the University of Massachusetts Medical School and Massachusetts DMH institutional review boards.

\section{Data Source}

This retrospective analysis identified pediatric member cases discussed by the PBHMI TCM workgroup with a peer-to-peer consultation between September 1, 2015, and August 28, 2016. The cases with completed outreach were logged in a deidentified tracking log. To assess prescriber satisfaction with the workgroup process, an anonymous and voluntary survey was faxed to prescribers who participated in a peer-to-peer consultation during the analysis period. Surveys were faxed again approximately 2 months later to increase response rate. Dichotomous survey responses were structured as either yes or no, while other assessment points used a 5-point Likert scale rating. Surveys were returned via fax or unmarked postal mail for confidentiality.

\section{Study Outcomes}

In cases where a peer-to-peer consultation was successfully completed, the outcome of the discussion (i.e., the documented details of the telephonic conversation between the psychiatrist and the prescriber tracked internally through the member case $\log$ ) was reviewed by the primary investigator and confirmed by the research team to determine the proportion of medication intervention recommendations that were accepted by the prescriber, modified and accepted, or rejected. MassHealth Pharmacy claims and subsequent PA resubmissions were reviewed for a minimum of 3 months following completion of peer-to-peer consultations to determine if proposed medication changes occurred as discussed during the prescriber outreach call. An intervention was considered to be accepted if a prescriber agreed to the recommendation from the peer-topeer consultation and evidence of the change was present in subsequent PA resubmissions and/or member pharmacy claims 


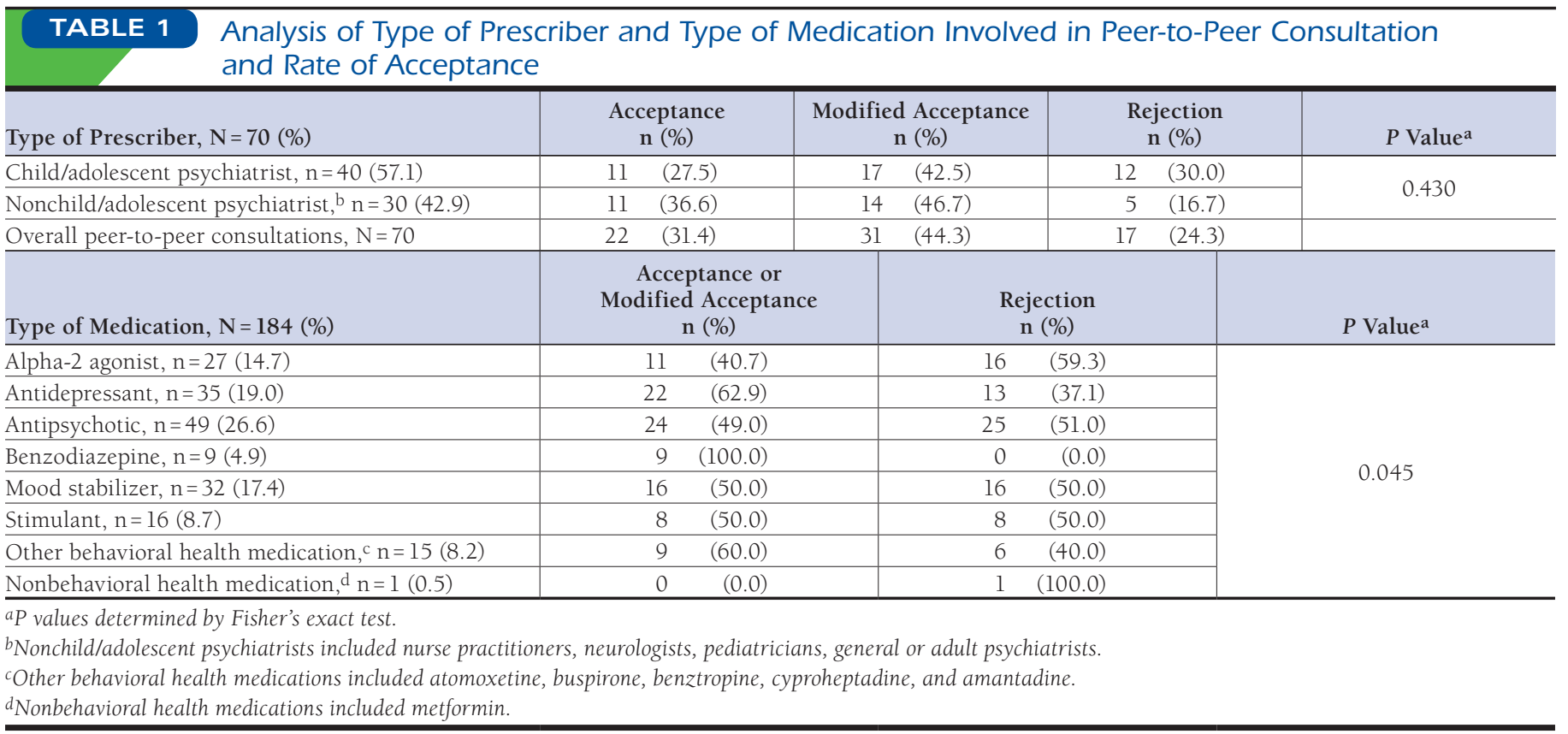

history at least 3 months after consultation. An intervention was considered to be modified and accepted if an aspect of the recommendation was implemented (i.e., decreased dose rather than discontinuing a medication) and evidence of the regimen change was present in the pharmacy claims database at least 3 months after consultation. Finally, an intervention was considered to be rejected if the prescriber verbally rejected the recommendation during the peer-to-peer consultation or the recommended change to the regimen was not evident with subsequent PA resubmissions and/or pharmacy claims history at least 3 months after consultation. Following the primary analysis, accepted, modified and accepted, and rejected recommendations were categorized to determine if there was any relation to the outcomes between the prescriber type or medication class.

\section{Analysis}

The primary analysis assessed the proportions of accepted, modified accepted, or rejected recommendations compared with the total number of peer-to-peer consultation discussions. To determine statistical significance between comparator groups for the secondary objective data, including prescriber type and medication class, the Fisher's exact test was used to test for differences between nominal variables for the prescriber type and the distribution of medication classes involved in the peer-to-peer consultation. Prescriber-type comparator groups included child adolescent psychiatrist versus nonchild adolescent psychiatrist based on specialty designation. Medication classes included alpha-2 agonists, antidepressants, antipsychotics, benzodiazepines, mood stabilizers, stimulants, other behavioral health medications, or other nonbehavioral health medications based on medications in a regimen with specific recommendations discussed during the consultation.

\section{Results}

\section{Primary Analysis}

During the evaluation period, 823 unique member cases were identified for PBHMI TCM workgroup review. After application of the high-risk algorithm (i.e., regimens containing $\geq 5$ medications, or cases with $<5$ medications including lithium, valproate, tricyclic antidepressants, benzodiazepines, antipsychotic use in a child aged $<13$ years, and/or antipsychotic polypharmacy), a total of 277 cases were selected for TCM discussion, of which 161 required a peer-to-peer consultation based upon clinical evaluation and case prioritization. A peer-to-peer outreach call was completed for 91 cases, 81 of which included a medication recommendation. A total of 70 cases were analyzed following application of the exclusion criteria, which included 11 cases with third-party coverage or coverage interruptions during the evaluation period. The time frame for investigation ranged from 4 months, for the most recently completed peer-to-peer consultation, to 17 months, for the earliest completed peer-to-peer consultation in the analysis period. The population age ranged from 5 to 17 years, with approximately $17.2 \%(12 / 70)$ of members aged 5 to 9 years, $41.4 \%$ (29/70) aged 10 to 13 years, and $41.4 \%$ (29/70) aged 14 to 17 years. The majority of members were male (57.1\%). Peer-to-peer consultation calls resulted in medication 
recommendations being accepted, modified accepted, and rejected in $31.4 \%(n=22), 44.3 \%(n=31)$, and $24.3 \%(n=17)$ of cases, respectively.

\section{Secondary Analyses}

Of the 70 completed peer-to-peer consultations, 40 were child/ adolescent psychiatrist prescribers and 30 were nonchild/adolescent psychiatrist prescribers (i.e., mid-level practitioners, pediatricians, neurologists, and general or adult psychiatrists). Peer-to-peer consultations completed with a child/adolescent psychiatrist corresponded with $27.5 \%$ (11/40), 42.5\% (17/40), and $30.0 \%(12 / 40)$ of recommendations being accepted, modified accepted, or rejected, respectively. Of the peer-to-peer consultations completed with a nonchild/adolescent psychiatrist, $36.6 \%(11 / 30)$ resulted in recommendation acceptance, $46.7 \%$ (14/30) were a modified acceptance, and 16.7\% (5/30) were rejected. The number of accepted recommendations offered during peer-to-peer consultations was not statistically significant $(P=0.43)$ when stratified by nonchild/adolescent prescribers and child/adolescent psychiatrists. Results are illustrated in Table 1.

Multiple recommendations were often provided during a single peer-to-peer consultation, with 184 individual medication recommendations provided during the time period evaluated. Changes to antipsychotic therapies were most frequently recommended $(26.6 \%, 49 / 184)$, followed by antidepressants (19.0\%, 35/184), mood stabilizers (17.4\%, 32/184), alpha-2 agonists $(14.7 \%, 27 / 184)$, stimulants $(8.7 \%, 16 / 184)$, other behavioral health medications $(8.2 \%, 15 / 184)$, benzodiazepines $(4.9 \%, 9 / 184)$, and nonbehavioral health medications $(0.5 \%, 1 / 184)$. Other behavioral health medications included atomoxetine, buspirone, benztropine, cyproheptadine, and amantadine. Nonbehavioral health medications included metformin. Medication recommendations pertaining to benzodiazepines had the highest percentage of prescriber acceptance or modified acceptance, with all recommendations accepted $(100 \%, 9 / 9)$, followed by antidepressants $(62.9 \%, 22 / 35)$, other behavioral health medications $(60.0 \%, 9 / 15)$, mood stabilizers $(50.0 \%, 16 / 32)$, stimulants $(50.0 \%, 8 / 16)$, antipsychotics (49.0\%, 24/49), and alpha-2 agonists (40.7\%, 11/27). Results are illustrated in Table 1.

The anonymous prescriber satisfaction survey was faxed to 52 unique prescribers who completed a peer-to-peer consultation during the evaluation period. Mid-level practitioners were excluded from the survey, as the target population was physician prescribers. A total of 24 surveys were returned, with 1 documenting the prescriber was no longer at the practice site. Of the 23 completed surveys included in the evaluation, 4 prescribers documented they did not recall the discussion for all survey questions. One prescriber documented he/she did not recall if the discussion was helpful, but answered the remaining survey questions. Overall, survey responses were variable. The majority of prescribers responded that peer-to- peer consultation did not lead to a change in their approach to managing the individual patient $(65.2 \%, 15 / 23)$ or their practice in general $(78.3 \%, 18 / 23)$. A total of $34.8 \%(8 / 23)$ of prescribers were satisfied overall with the PBHMI TCM workgroup outreach process, and 39.1\% (9/23) prescribers responded the consultation was helpful. These results and additional responses are illustrated in Figure 1.

\section{Discussion}

The MassHealth PBHMI program and TCM workgroup is a novel approach in managing the high rates of behavioral health medication utilization in the pediatric population. This analysis is the first to attempt to assess the outcomes of a prescriber peer-to-peer consultation program. To our knowledge, no other state Medicaid programs have implemented a similar process.

During the analysis period, peer-to-peer consultation resulted in a $75.7 \%(53 / 70)$ overall acceptance or modified acceptance rate by prescribers. The small sample size in this observational evaluation and lack of a defined control group prevented direct associations between the endpoints and outcomes. Further research is required to determine if prescriber specialty and medication class may be influencing factors on recommendation acceptance.

While benzodiazepines did not account for a large volume of individual recommendations, this medication class had the highest acceptance rate with all recommendations accepted and implemented by the prescribers. This observation may be reflective of prescribers considering that benzodiazepine agents are high risk or have low clinical benefit. Additionally, benzodiazepine agents may have been indicated for short-term use and therefore, discontinued as a part of the prescriber's original treatment plan.

Results of the prescriber survey to assess the overall satisfaction and perceived value of the peer-to-peer consultation process were variable, with no significant findings. Of the 23 respondents, 8 found the amount of time required for the peer-to-peer consultation to be valuable and expressed overall satisfaction with the consultation process; however, 9 were not satisfied with the consultation scheduling process, which identified potential opportunities for process improvement. The prescriber survey responses did not correlate with our findings that $75.7 \%$ of peer-to-peer consultations resulted in the implementation of at least 1 recommendation following a peerto-peer consultation. This may be due to recall bias caused by a delay in recommendation, implementation, or the time lapse between the peer-to-peer consultation and survey completion. Future attempts at eliciting detailed and accurate responses should ideally occur at a time closer to the completion of the peer-to-peer consultation, which may also potentially increase response rate. The survey may have also been used to offer dissatisfaction with the initiative rather than the peer-to-peer consultation process specifically. 


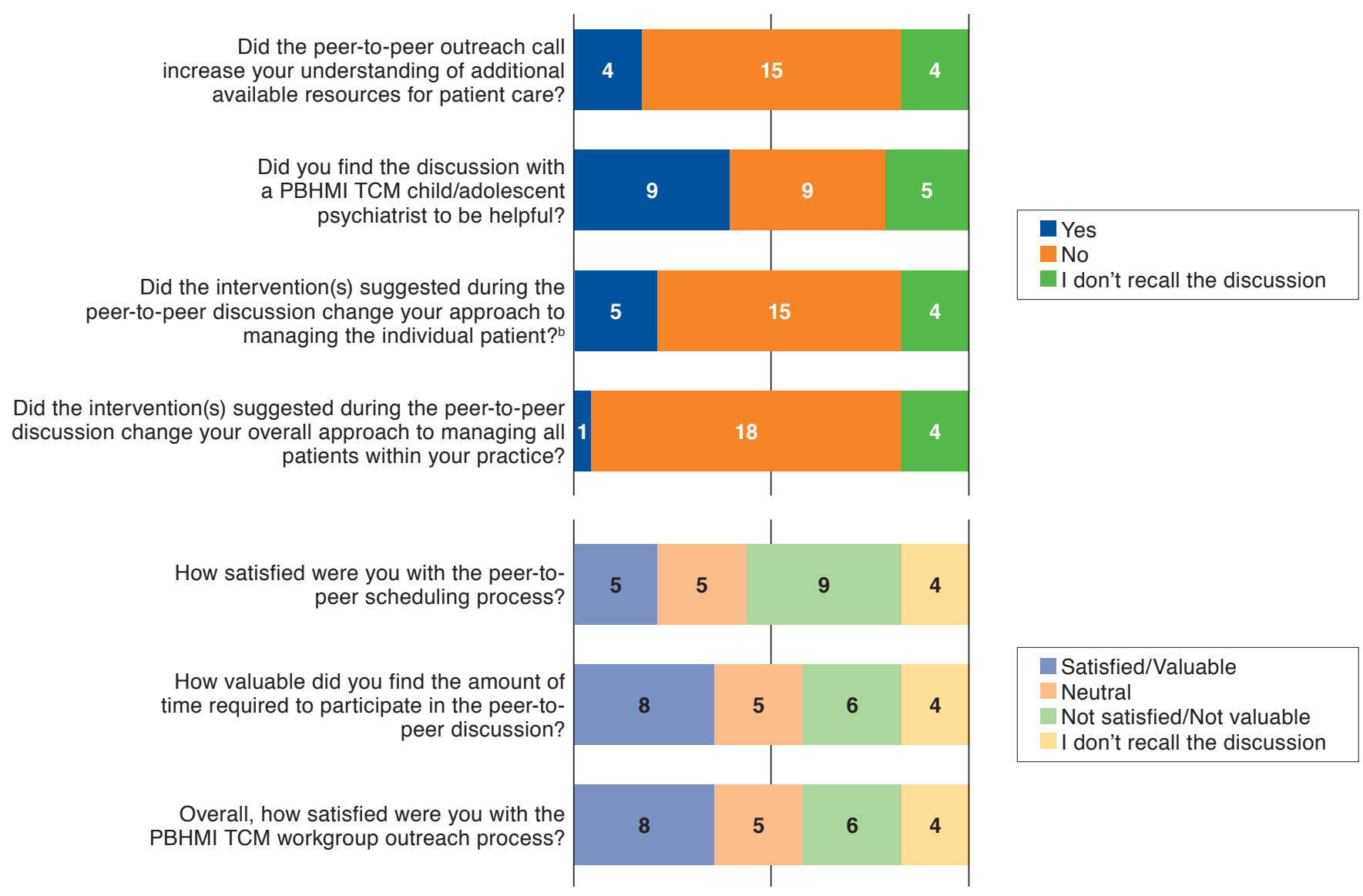

aThe sum of the responses to each question is 23 , as 23 prescribers responded to the survey.

"One prescriber responded both "yes" and "no," likely due to participation in more than 1 peer-to-peer consultation. PBHMI=Pediatric Behavioral Health Medication Initiative; TCM = therapeutic class management.

\section{Limitations}

Findings in this analysis are subject to several limitations. Changes that were made to a member's regimen following a peer-to-peer consultation may not be fully reflected by data collected from the pharmacy claims database or information submitted by prescribers on PA submission forms. Acceptance of recommendations may have been limited by additional factors, including a member changing providers, multiple providers involved in care, patient setting, or a decline in mental health status, which may not have been provided at the time of the peer-to-peer consultation. Cases in which a peer-topeer consultation occurred earlier in the analysis period were followed for a longer duration, and therefore, allowed more time for interventions to take place compared with those completed toward the end of the analysis period. Determination of consultation recommendation for acceptance, modified acceptance, and rejection was based on pharmacy claims data alone. Therefore, it is possible that the results for recommendation acceptance were overestimated, as recommended changes may have been part of the prescriber's original treatment plan versus being a result of the workgroup's intervention.

The PBHMI workgroup routinely reviews cases for members with third-party insurance coverage; however, a lack of access to the primary payer's pharmacy claims for these members prevented inclusion for this analysis, and therefore, may not represent the full effect of the PBHMI TCM workgroup interventions. Dataset limitations included incomplete information regarding member race/ethnicity, socioeconomic status, and/or other potential covariates. In addition, the sample size was not large enough to identify statistically significant differences in the outcomes analyzed. 
The prescriber survey yielded a low response rate as it was administered 17 months following the earliest completed peer-to-peer consultation in the analysis period. Recall bias may have affected the survey results due to the extended time period between the outreach and survey administration. In addition, inclusion of mid-level practitioners as respondents for future surveys may also improve generalizability of outcomes. As the survey was anonymous, there was no validated process or means to verify who completed the survey (e.g., prescriber versus office staff). The prescriber survey was distributed on 2 separate occasions in efforts to increase the response rate, and duplicate anonymous survey submissions may have occurred.

\section{Conclusions}

The development and implementation of a multidisciplinary PBHMI TCM workgroup can serve as a proactive approach for identifying complex pediatric cases involving polypharmacy and other potential safety concerns. The small sample size in this observational evaluation and lack of a defined control group prevented direct associations between the endpoints and outcomes. Further research is required to determine if prescriber specialty, medication class, or other influencing factors may have an effect on the acceptance of peer-to-peer outreach recommendations.

\section{Authors}

ASHLEY N. CHIARA, PharmD, MBA; NEHA KASHALIKAR, PharmD; THOMAS C. POMFRET, PharmD, MPH, BCPS; and PATRICIA LETO, PharmD, Clinical Pharmacy Services, University of Massachusetts Medical School, Shrewsbury. MICHAEL C. ANGELINI, MA, PharmD, BCPP, Clinical Pharmacy Services, University of Massachusetts Medical School, Shrewsbury, and MCPHS University, Boston. KIMBERLY LENZ, PharmD, and PAUL L. JEFFREY, PharmD, MassHealth, Quincy, Massachusetts. DONNA M. FABER, PharmD, BCPS, BCPP, Phoenix Veterans Affairs Health Care System, Phoenix, Arizona. JOEL GOLDSTEIN, MD, DCF/DMH, Caring Together Initiative, Boston, Massachusetts, and Department of Psychiatry, Cambridge Health Alliance, Harvard Medical School, Cambridge, Massachusetts. KAREN M. CLEMENTS, ScD, Center for Health Policy and Research, University of Massachusetts Medical School, Shrewsbury.

AUTHOR CORRESPONDENCE: Thomas C. Pomfret, PharmD, MPH, BCPS, Clinical Consultant Pharmacist Team Lead, Clinical Pharmacy Services, UMass Medical School, 333 South St., Shrewsbury, MA 01545-7807. Tel.: 774.455.3238;

E-mail: Thomas.Pomfret@umassmed.edu.

\section{DISCLOSURES}

No outside funding supported this study. The authors have nothing to disclose.

A poster of this project was presented at the AMCP Managed Care \& Specialty Pharmacy Annual Meeting 2017; March 27-30, 2017; in Denver, CO.

\section{REFERENCES}

1. Comer JS, Olfson M, Mojtabai R. National trends in child and adolescent psychotropic polypharmacy in office-based practice, 1996-2007. J Am Acad Child Adolesc Psychiatry. 2010;49(10):1001-10.

2. APA Working Group on Psychoactive Medication for Children and Adolescents. Report of the Working Group on Psychoactive Medications for Children and Adolescents. Psychopharmacological, psychosocial, and combined interventions for childhood disorder: evidence base, contextual factors, and future directions. American Psychological Association. 2006 Available at: http://www.apa.org/pi/families/resources/child-medications. pdf. Accessed June 7, 2018

3. American Academy of Child and Adolescent Psychiatry. Practice parameter on the use of psychotropic medication in children and adolescents. J Am Acad Child Adolesc Psychiatry. 2009;48(9):961-73.

4. American Academy of Child and Adolescent Psychiatry. Practice parameter for psychodynamic psychotherapy with children. J Am Acad Child Adolesc Psychiatry. 2012;51(5):541-57.

5. U.S. Government Accountability Office. Children's mental health: concerns remain about appropriate services for children in Medicaid and foster care. GAO-13-15. December 2012. Available at: https://www.gao.gov/ assets/660/650716.pdf. Accessed June 7, 2018.

6. U.S. Government Accountability Office. Foster children: HHS guidance could help states improve oversight of psychotropic prescriptions. GAO-12201. December 2011. Available at: https://www.gao.gov/assets/590/586906. pdf. Accessed June 7, 2018

7. Jureidini J, Tonkin A, Jureidini E. Combination pharmacotherapy for psychiatric disorders in children and adolescents: prevalence, efficacy, risks and research need. Paediatr Drugs. 2013;15(5):377-91.

8. Kreider AR, Matone M, Bellonci C, et al. Growth in the concurrent use of antipsychotics with other psychotropic medications in Medicaid-enrolled children. J Am Acad Child Adolesc Psychiatry. 2014;53(9):960-70.e2.

9. Chen H, Patel A, Sherer J, Aparasu R. The definition and prevalence of pediatric psychotropic polypharmacy. Psychiatr Serv. 2011;62(12):1450-55.

10. Zito JM, Safer DJ, Sai D, et al. Psychotropic medication patterns among youth in foster care. Pediatrics. 2008;121(1):e157-63.

11. dosReis S, Zito JM, Safer DJ, Gardner JF, Puccia KB, Owens PL. Multiple psychotropic medication use for youths: a two-state comparison. J Child Adolesc Psychopharmacol. 2005;15(1):68-77.

12. Fontanella CA, Warner LA, Phillips GS, Bridge JA, Campo JV. Trends in psychotropic polypharmacy among youths enrolled in Ohio Medicaid, 2002-2008. Psychiatr Serv. 2014;65(11):1332-40.

13. Zito JM, Burcu M, Ibe A, Safer DJ, Magder LS. Antipsychotic use by Medicaid-insured youths: impact of eligibility and psychiatric diagnosis across a decade. Psychiatr Serv. 2013;64(3):223-29.

14. Hilt RJ, Chaudhari M, Bell JF, Wolf C, Koprowicz K, King BH. Side effects from use of one or more psychiatric medications in a populationbased sample of children and adolescents. J Child Adolesc Psychopharmacol. 2014;24(2):83-89. 